\title{
Association between preoperative hemoglobin and postoperative moderate and severe anemia among patients undergoing primary total knee arthroplasty: a single-center retrospective study
}

Guorui Cao' ${ }^{1}$ Xiuli Yang ${ }^{2}$, Hong Xu², Chen Yue1, Zeyu Huang ${ }^{2}$, Shaoyun Zhang ${ }^{3}$, Songtao Quan ${ }^{1}$, Junna Yao ${ }^{1}$, Minglu Yang ${ }^{1}$ and Fuxing Pei ${ }^{2^{*}}$

\begin{abstract}
Background: Postoperative moderate and severe anemia (PMSA) has been a serious perioperative complication in primary total knee arthroplasty (TKA). However, the ideal cutoff values to predict PMSA is still undetermined. The aim of this study was (1) to identify the risk factors associated with PMSA and (2) to establish the cutoff values of preoperative hemoglobin (HB) associated with increased PMSA in primary TKA.

Methods: We identified 474 patients undergoing primary TKA and separated those in which PMSA (HB was less than $110 \mathrm{~g} / \mathrm{L}$ on postoperative day 1 and 3) was developed from those without PMSA. Multivariate logistic regression model was used to identify independent risk factors for PMSA. Area under the receiver-operator curve (AUC) was used to determine the best-supported preoperative HB cutoff across all the patients.

Results: The PMSA rate in primary TKA was 53.2\%. Significant risk factors were lower preoperative HB (OR [odds ratio] $=1.138,95 \% \mathrm{Cl}$ [confidence interval] $=1.107-1.170, p<0.001)$ and more intraoperative blood loss $(\mathrm{OR}=1.022$, $95 \% \mathrm{Cl} 1.484-4.598, p<0.001)$.

A preoperative HB cutoff value that maximized the AUC was $138.5 \mathrm{~g} / \mathrm{L}$ for men (sensitivity: $79.4 \%$, specificity: $75.0 \%$ ) and $131.5 \mathrm{~g} / \mathrm{L}$ for women (sensitivity: $74.7 \%$, specificity: $80.5 \%$ ), respectively.

Conclusion: We should recognize and consider the related risk factors to establish specific, personalized risk assessment for PMSA, including preoperative HB and intraoperative blood loss. Of these, preoperative HB was a referable tool to predict PMSA in primary TKA.
\end{abstract}

Keywords: Moderate and severe anemia, Risk factors, Total knee arthroplasty, Preoperative hemoglobin

\footnotetext{
*Correspondence: peifuxingwestchina@163.com

${ }^{2}$ Department of Orthopaedic Surgery, West China Hospital, Sichuan University, No. 87 Guoxue Road, Chengdu 610041, Sichuan Province, People's Republic of China

Full list of author information is available at the end of the article
}

\section{Introduction}

Total knee arthroplasty (TKA) is an effective treatment to correct deformity, relieve pain and improve quality of life for advanced osteoarthritis of the knee [1]. Although the application of enhanced recovery protocols, especially the use of tranexamic acid (TXA), dramatically reduced perioperative blood loss and transfusion following permits use, sharing, adaptation, distribution and reproduction in any medium or format, as long as you give appropriate credit to the original author(s) and the source, provide a link to the Creative Commons licence, and indicate if changes were made. The images or other third party material in this article are included in the article's Creative Commons licence, unless indicated otherwise in a credit line to the material. If material is not included in the article's Creative Commons licence and your intended use is not permitted by statutory regulation or exceeds the permitted use, you will need to obtain permission directly from the copyright holder. To view a copy of this licence, visit http://creativecommons.org/licenses/by/4.0/. The Creative Commons Public Domain Dedication waiver (http://creativeco mmons.org/publicdomain/zero/1.0/) applies to the data made available in this article, unless otherwise stated in a credit line to the data. 
primary TKA, postoperative moderate and severe anemia (PMSA) has been still a frequent and troublesome issue $[2,3]$. PMSA was always associated with longer length of stay (LOS), higher readmission risk and increased risk of complications and morbidity, such as transfusion, joint infection, bleeding events, kidney injury and even death [4-7]. Given the increasing number of TKAs performed around the world, an important concern for surgeons was how to minimize the risk of PMSA in primary TKA [8].

To our best knowledge, no previous study has identified the specific risk factors associated with PMSA for primary TKA. Considering that preoperative hemoglobin (HB) was a strong predictor for transfusion after TKA, one possible predictor for PMSA was preoperative $\mathrm{HB}$ $[9,10]$. Currently, the risk factor for PMSA, the sensitive and specific preoperative $\mathrm{HB}$ value for predicting PMSA following primary TKA have not been determined. Therefore, we perform a retrospective cohort study to investigate (1) what are the risk factors associated with PMSA? (2) If preoperative HB is highly associated with PMSA, what is appropriate cutoff values of preoperative $\mathrm{HB}$ for PMSA in primary TKA?

\section{Materials and methods}

\section{Patients and design}

This study was a single-center retrospective cohort study and approved by the hospital's Institutional Review Board. The patients undergoing primary TKA owing to osteoarthritis and rheumatoid arthritis were included from October 2015 to August 2018 in our institution. We excluded the patients with any of the following situations: cardiovascular problems, history of deep venous thrombosis (DVT) or pulmonary embolism (PE) and incomplete medical records.

The blood samples were tested preoperatively, on postoperative (POD) 1 and POD3. Moderate anemia was defined as a HB level between 80 and $110 \mathrm{~g} / \mathrm{L}$ and severe anemia was defined as a $\mathrm{HB}$ level $<80 \mathrm{~g} / \mathrm{L}$ for both genders [11]. Because of small number of severe anemia $(n=5)$, patients with moderate and severe anemia were analyzed together. The enrolled patients were divided into PMSA and non-PMSA groups according to level of postoperative hemoglobin (HB) on POD1 or POD3.

\section{Surgical technique}

All the surgeries were conducted by the same senior surgeon under general anesthesia. The operations were performed through a midline skin incision, medial parapatellar approach and a measured resection technique. The prosthesis was cemented posterior-stabilized prosthetic design with patellar resurfacing. Controlled hypotension was induced with the blood pressure being maintained 90-110 $\mathrm{mmHg} / 60-70 \mathrm{mmHg}$ during operation. No tourniquet, nerve block, intravenous patient-controlled analgesia or blood salvage system were used.

\section{Perioperative protocol}

All the patients received enhanced recovery protocols [12]. The physical prophylaxis and chemoprophylaxis were applied to prevent DVT and PE. Physical prophylaxis included the exercises of ankle pump and knee extension, and the application of intermittent pneumatic compression device early postoperatively. Chemoprophylaxis included the application of low-molecular-weight heparin (2000 IU in $0.2 \mathrm{ml}$; Clexane, Sanofi-Aventis, France) or $10 \mathrm{mg}$ Rivaroxaban (Xarelto, Bayer, Germany) 6-8 h postoperatively, repeating once a day for 14 days. Doppler ultrasound examinations were used routinely to detect DVT preoperatively, on POD3 and POD14.

The blood management strategies were implemented on the basis of our previous studies $[13,14]$. The TXA $(20 \mathrm{mg} / \mathrm{kg}$ ) was administered intravenously $5-10 \mathrm{~min}$ before skin incision, along with $1 \mathrm{~g}$ intravenous TXA (diluted in $100 \mathrm{~mL}$ of normal saline solution) at 3, 6, 12 and $24 \mathrm{~h}$ postoperatively. Patients received the combination of 10,000 IU of erythropoietin subcutaneously once a day for 5 days and $200 \mathrm{mg}$ of intravenous iron supplements once every other day when the perioperative $\mathrm{HB}$ was less than $110 \mathrm{~g} / \mathrm{L}$, and only $300 \mathrm{mg}$ of oral iron supplements every day when the level of $\mathrm{Hb}$ was 110 $130 \mathrm{~g} / \mathrm{L}$ for males and $110-120 \mathrm{~g} / \mathrm{L}$ for females. Transfusions were applied when the $\mathrm{HB}$ value was $<70 \mathrm{~g} / \mathrm{L}$ or 70-100 g/L with symptoms of anemia, such as altered mental status, palpitations or decreased exercise tolerance [15].

\section{Outcome measurements}

Demographic characteristics and comorbidities, including age, gender, diagnosis, body mass index, incidence of hypertension, diabetes, coronary heart disease and so on were collected. Laboratory markers $(\mathrm{Hb}$, hematocrit (Hct), erythrocyte sedimentation rate, C-reactive protein and interleukin 6) were tested preoperatively, on POD1 and POD3. In addition, American Society of Anesthesiologists physical status classification, the application of TXA and drainage, operative time, intraoperative blood loss, allogenic transfusion units and rate, length of stay (LOS) after surgery, total expenses and postoperative complications were carefully recorded. Wound complication were defined and classified on the basis of previous study [16].

\section{Statistical analyses}

All analyses were performed using SPSS version 22.0 (SPSS Inc., USA) and EmpowerStats (http://www.empow erstats.com/). Descriptive and univariate analyses were 
initially conducted using SPSS software. Excluding variables with significant correlation, statistically significant or close to statistically significant variables were enrolled a multivariable logistic regression model to examine independent risk factor for PMSA in primary TKA. Receiver operating characteristic (ROC) curve and curve fitting were performed by EmpowerStats software. Youden's index was applied to determine the optimal predictive cutoffs for preoperative HB. Independent $t$-test was used for continuous variables and chi-squared test or Fisher's exact test for categorical and dichotomous variables. A $p$ value of $<0.05$ was considered significant.

\section{Results}

Five hundred and twenty-five patients were included initially. Fifty-one patients were excluded because of incomplete medical records or inconformity to inclusion criteria. Eventually, 474 patients were enrolled, including 252 patients with PMSA and 222 patients without PMSA (Fig. 1). Totally, the prevalence of PMSA was $53.2 \%$ for primary TKA, 12 cases $(12 / 252,5.2 \%)$ developed anemia on POD1 and 239 cases (239/252, 94.8\%) on POD3. In addition, only $5(5 / 252,2.0 \%)$ cases were severe anemia which was entirely occurred on POD3 and other were moderate anemia.

Baseline characteristics of PMSA and non-PMSA patients are shown in Table 1. The risk factors for PMSA after univariate analyses were female, lower body mass index (BMI), diagnosis as rheumatoid arthritis, preoperative anemia, lower level of preoperative $\mathrm{HB}$ and Hct, more intraoperative blood loss. Besides, the application of TXA was a protective factor $(p<0.05)$.

Risk factors independently associated with PMSA after logistic regression included more intraoperative blood loss (OR [odds ratio] $=1.022,95 \%$ CI [confidence interval $])=1.484-4.598, p<0.001)$ and lower preoperative $\mathrm{Hb}$ $(\mathrm{OR}=1.138,95 \%$ CI 1.107-1.170, $p<0.001)$ (Table 2).

Preoperative $\mathrm{HB}$ was found to be the variable most highly associated with PMSA for men (AUC, 0.796) and women (AUC, 0.825), respectively. Moreover, the value of AUC following bootstrap smooth ROC was 0.781 and 0.808 separately (Fig. 2). The optimal predictive cutoff of preoperative HB for PMSA was $138.5 \mathrm{~g} / \mathrm{L}$ (sensitivity: $79.4 \%$, specificity: $75.0 \%$ ) in male and $131.5 \mathrm{~g} / \mathrm{L}$

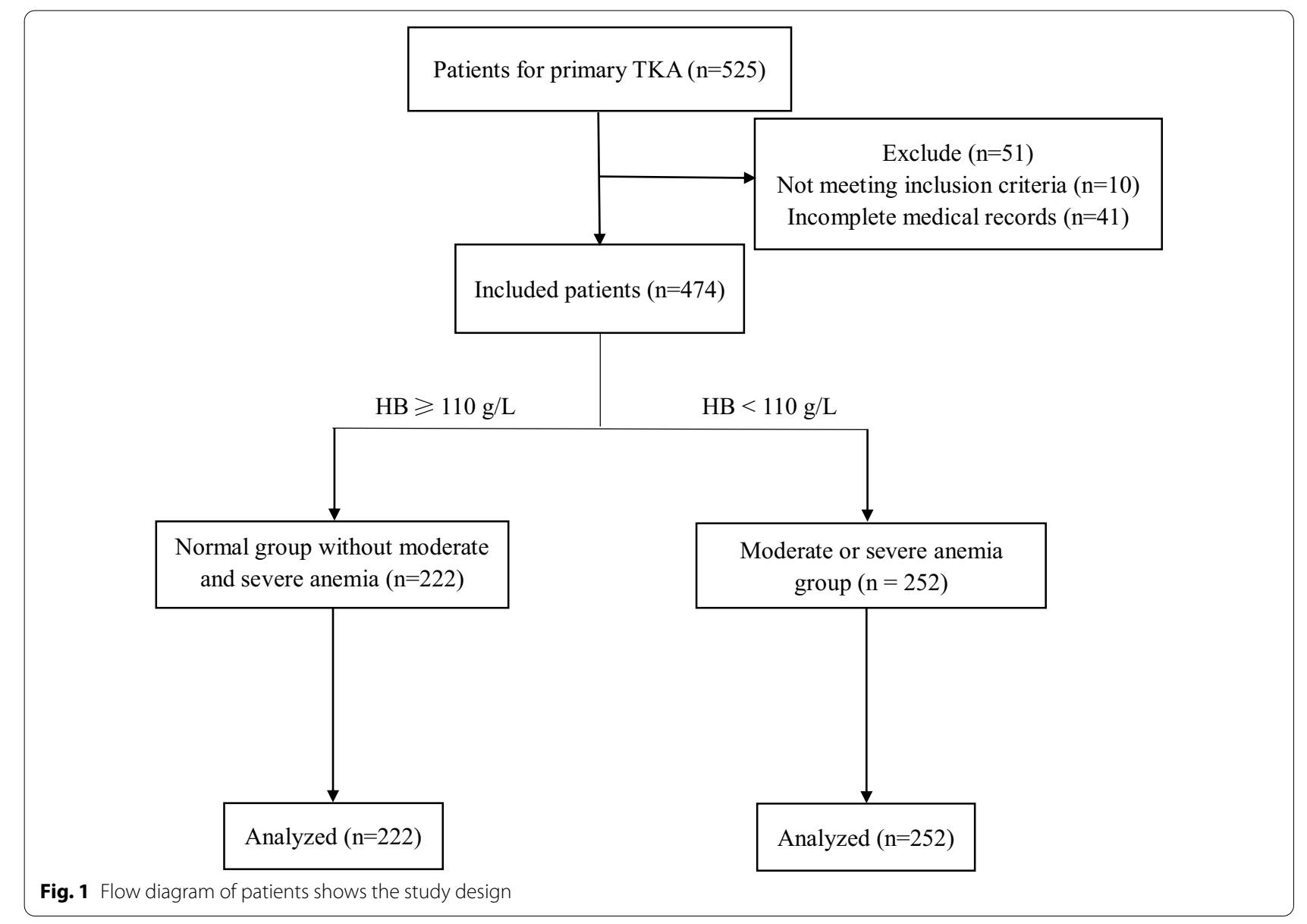


Table 1 Baseline of patients with moderate and severe anemia after primary TKA

\begin{tabular}{|c|c|c|c|}
\hline Baseline characteristic & Non-DMSA group, $N=222$ & DMSA group, $N=252$ & $p$ \\
\hline \multicolumn{4}{|l|}{ Demographic characteristics } \\
\hline $\operatorname{Age}(\bar{X} \pm S)$ & $66.8 \pm 7.8$ & $66.2 \pm 7.9$ & 0.484 \\
\hline Female $(N, \%)$ & $154(69.4 \%)$ & $236(93.7 \%)$ & $<0.001^{*}$ \\
\hline $\operatorname{BMI}\left(\bar{X} \pm S, \mathrm{~kg} / \mathrm{m}^{2}\right)$ & $26.4 \pm 3.5$ & $25.4 \pm 3.7$ & $0.003^{*}$ \\
\hline Diagnosis (RA/OA) & $1 / 221$ & $12 / 240$ & $0.004^{*}$ \\
\hline Preoperative knee ROM $\left(^{\circ}\right)$ & $99.0 \pm 17.2$ & $100.0 \pm 15.1$ & 0.480 \\
\hline Preoperative HSS & $49.9 \pm 8.3$ & $48.9 \pm 8.4$ & 0.275 \\
\hline \multicolumn{4}{|l|}{ Comorbidities (N, \%) } \\
\hline Hypertension & $113(50.9 \%)$ & $138(54.8 \%)$ & 0.401 \\
\hline Diabetes & $31(14.0 \%)$ & $41(16.3 \%)$ & 0.485 \\
\hline Coronary heart disease & $15(6.8 \%)$ & $16(6.3 \%)$ & 0.858 \\
\hline Preoperative anemia & $6(6.8 \%)$ & $46(18.3 \%)$ & $<0.001^{*}$ \\
\hline \multicolumn{4}{|l|}{ Preoperative laboratories } \\
\hline $\mathrm{HB}(\mathrm{g} / \mathrm{L})$ & $140.2 \pm 11.0$ & $125.1 \pm 10.2$ & $<0.001^{*}$ \\
\hline Hct & $42.0 \pm 3.1$ & $38.3 \pm 3.1$ & $<0.001^{*}$ \\
\hline $\operatorname{ESR}(\mathrm{mm} / \mathrm{h})$ & $21.3 \pm 20.2$ & $22.4 \pm 17.8$ & 0.116 \\
\hline CRP (mg/L) & $3.50 \pm 2.77$ & $3.77 \pm 3.67$ & 0.372 \\
\hline IL-6 (mg/L) & $3.8 \pm 2.4$ & $4.3 \pm 4.2$ & 0.126 \\
\hline \multicolumn{4}{|l|}{ Operative variables } \\
\hline ASA class $(N, \%)$ & & & 0.959 \\
\hline $1-2$ & $174(78.4 \%)$ & $198(78.6 \%)$ & \\
\hline$\geq 3$ & $48(21.6 \%)$ & $54(21.4 \%)$ & \\
\hline Drainage use $(N, \%)$ & $42(18.9 \%)$ & $66(26.2 \%)$ & 0.060 \\
\hline TXA use $(N, \%)$ & $217(97.7 \%)$ & $226(89.7 \%)$ & $<0.001^{*}$ \\
\hline Operative time (min) & $76.4 \pm 12.1$ & $77.7 \pm 15.5$ & 0.340 \\
\hline Intraoperative blood loss (mL) & $110.0 \pm 22.8$ & $130.9 \pm 34.7$ & $<0.001^{*}$ \\
\hline
\end{tabular}

BMI, body mass index; RA, rheumatoid arthritis; $\mathrm{OA}$, osteoarthritis; ROM, range of motion; HSS, hospital for special surgery knee score; HB, hemoglobin; $\mathrm{Hct}$, hematocrit; ESR, erythrocyte sedimentation rate; CRP, C reaction protein; IL-6, interleukin-6; ASA, American Society of Anesthesiologists; TXA, Tranexamic acid $p$ value calculated using independent $t$-test, Pearson's chi-squared test or Fisher's exact test

${ }^{*}$ Significant difference

Table 2 Logistic regression models for moderate and severe anemia after primary TKA

\begin{tabular}{lllc}
\hline Parameter & Odds ratio & $\begin{array}{l}\mathbf{9 5 \%} \\
\text { Confidence } \\
\text { interval }\end{array}$ & $p$ \\
\hline Female & 1.931 & $0.928-4.015$ & 0.078 \\
BMI & 1.033 & $0.967-1.104$ & 0.340 \\
Diagnosis, RA & 3.171 & $0.353-28.456$ & 0.303 \\
Preoperative Hb (g/L) & 1.138 & $1.107-1.170$ & $<0.001^{*}$ \\
Drainage use & 1.354 & $0.734-2.499$ & 0.333 \\
TXA use & 0.358 & $0.085-1.506$ & 0.161 \\
Intraoperative blood loss & 1.022 & $1.010-1.034$ & $<0.001^{*}$ \\
\hline
\end{tabular}

$\mathrm{BMI}$, body mass index; RA, rheumatoid arthritis; $\mathrm{Hb}$, hemoglobin; TXA, Tranexamic acid

$p$ value calculated using multivariate logistic regression

* Significant difference (sensitivity: $74.7 \%$, specificity: $80.5 \%$ ) in female sex (Table 3). As expected, the risk of PMSA in primary TKA decreased when the preoperative $\mathrm{HB}$ increase for men and women (Fig. 3).

The situations of LOS, expenses and related complication are summarized in Table 4. The LOS in PMSA group was longer than that in non-PMSA group $(3.92 \pm 0.94$ vs $3.06 \pm 0.92, p=0.002)$. The similar trend was shown in hospitalization expenses $(59,532.3 \pm 9618.2$ vs $57,143.1 \pm 9246.5, p=0.031)$. In addition, the incidence of complications, including transfusion, DVT, PE, wound complications and so on did not reach statistical difference between the two groups $(p>0.05)$.

\section{Discussion}

Anemia was a common complication after orthopedic surgery, resulting in a series of problems. Generally, mild anemia could be corrected by oral iron supplements, reinforced nutrition. By comparison, PMSA was usually 


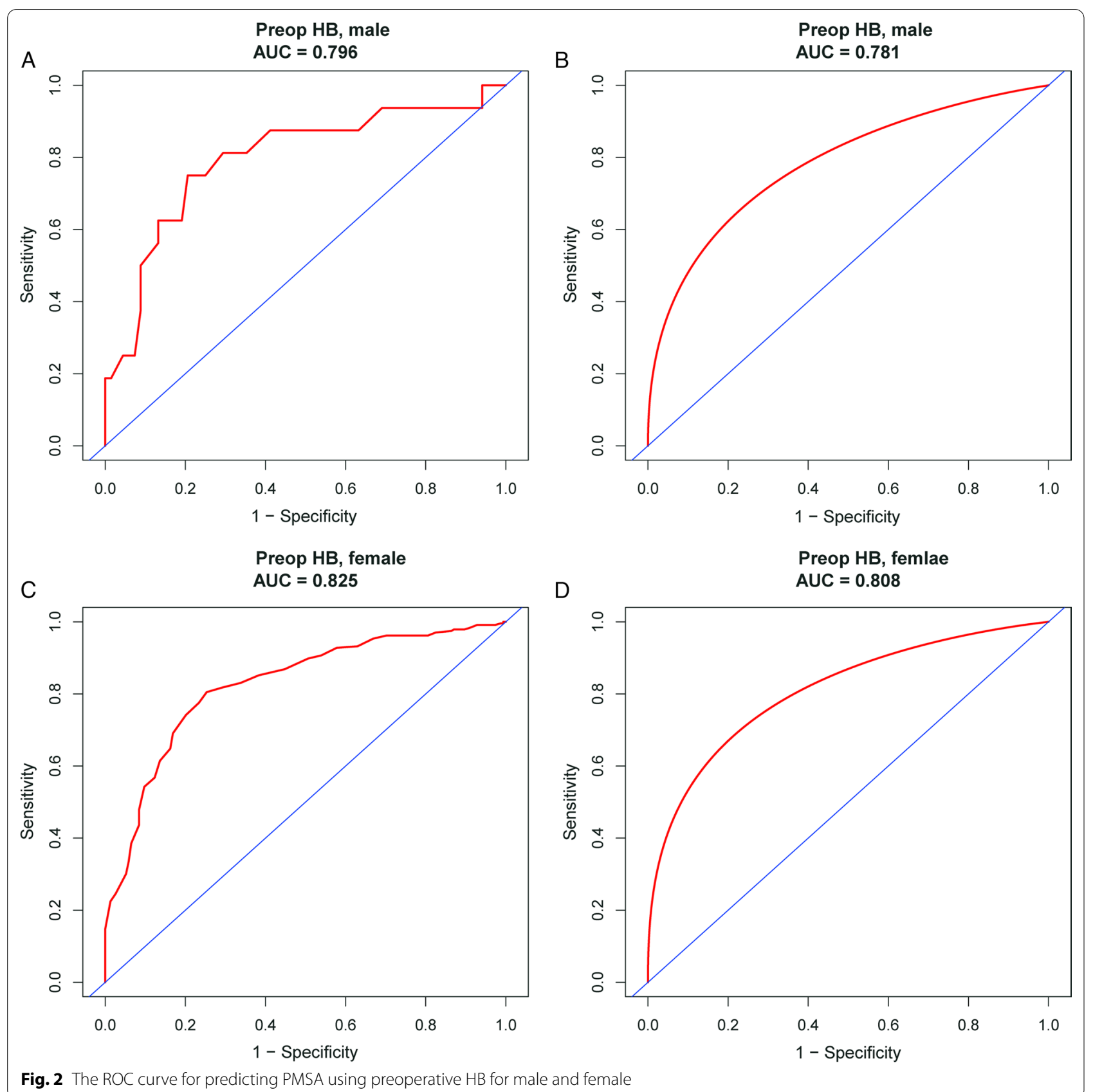

Table 3 The diagnostic value of preoperative HB for PMSA

\begin{tabular}{lllllll}
\hline Variables & Youden's index & \multicolumn{2}{l}{ Preoperative } & & \multicolumn{2}{l}{ Maximize } \\
& & Cutoff & AUC & & Sensitivity (\%) & Specificity (\%) \\
\hline Male & 0.544 & $138.5(\mathrm{~g} / \mathrm{L})$ & $0.796 / 0.781$ & 79.4 & 75.0 \\
Female & 0.552 & $131.5(\mathrm{~g} / \mathrm{L})$ & $0.825 / 0.808$ & & 74.7 & 80.5 \\
\hline
\end{tabular}

$\mathrm{HB}$, hemoglobin; PMSA, postoperative moderate or severe anemia; AUC, Area under the receiver operating characteristics curve 

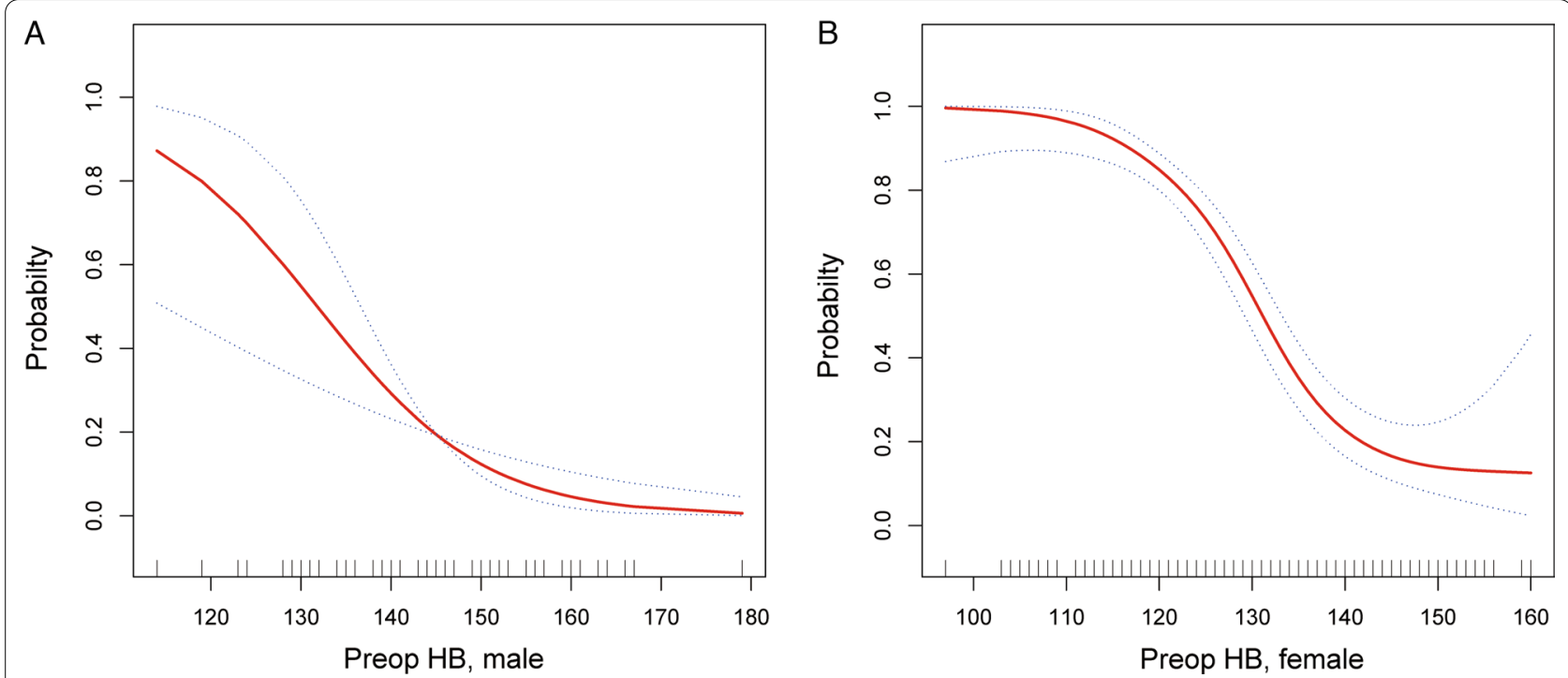

Fig. 3 The predictive probability of PMSA by preoperative HB adjusted for sex

Table 4 LOS, expenses and complications

\begin{tabular}{llll}
\hline Variables & Non-DMSA group, $\boldsymbol{N = 2 2 2}$ & DMSA group, $\boldsymbol{N = 2 5 2}$ & $\boldsymbol{p}$ \\
\hline LOS & $3.06 \pm 0.92$ & $3.92 \pm 0.94$ & $0.002^{*}$ \\
Hospitalization expenses $\Delta$ & $57,143.1 \pm 9246.5$ & $59,532.3 \pm 9618.2$ & $0.031^{*}$ \\
Transfusion & 0 & $2(0.8 \%)$ & 0.501 \\
Death & 0 & 0 & - \\
DVT & $2(0.9 \%)$ & $1(0.4 \%)$ & 0.602 \\
PE & 0 & $29(11.5 \%)$ & - \\
Nausea and vomiting & $17(7.7 \%)$ & 0 & 0.158 \\
Cardiac infarction & $1(0.5 \%)$ & 0 & - \\
Stroke & 0 & 0 & - \\
Acute renal failure & 0 & $29(11.5 \%)$ & 0.468 \\
Wound complications & $23(10.4 \%)$ & $2(0.8 \%)$ & 0.690 \\
Readmission & $2(0.9 \%)$ & & 1.000 \\
\hline
\end{tabular}

Wound complications included exudation, bleeding, swelling, infection and impaired wound healing

LOS, length of stay; DVT, deep venous thrombosis; PE, pulmonary embolism

$p$ value calculated using independent $t$-test, Pearson's chi-squared test or Fisher's exact test

$\Delta$ Results were presented as Chinese yuan

${ }^{*}$ Significant difference

difficult to resolve, needing further treatments, such as intravenous iron, erythropoietin, even transfusion [17, 18]. Moreover, patients who developed PMSA had higher risk of developing postoperative complications relative to patients with mild anemia following joint replacement [19]. Thus, we selected PMSA as the outcome variable, evaluating related risk factor and attempting to decrease the rate.

Current literature is mainly limited to describe the effect of preoperative anemia on the transfusion, LOS, complication, functional and quality of life outcomes in the setting of primary TKA [19-21]. As preoperative anemia, postoperative anemia is also an independent risk factor for delayed postoperative rehabilitation, increasing complications and readmission rates $[4,5,7]$. Our study showed that preoperative $\mathrm{HB}$ and intraoperative blood loss are significantly associated with increased PMSA. The ideal preoperative HB cutoff value was $131.5 \mathrm{~g} / \mathrm{L}$ for males and $138.5 \mathrm{~g} / \mathrm{L}$ for females in primary TKA. Identifying a robust preoperative $\mathrm{HB}$ cutoff could offer 
opportunities for doctors to treatment patient's anemia and decrease the PMSA risk. Patients whose preoperative $\mathrm{HB}$ was less than the cutoff value could receive intervention early, such as erythropoietin and iron supplementation, to avoid PMSA and associated complications [22-27].

Currently, there is a lack of consensus and evidencebased practice when it comes to the risk factors for PMSA in patients undergoing TKA. However, the risk factor for transfusion in TKA was extensively studied. The researchers concluded that lower level of preoperative $\mathrm{HB}$ and increased amount of intraoperative blood loss were independently associated with transfusion in TKA [28-30]. Considering the trigger of transfusion and diagnosis of PMSA was both mainly decided by the level of postoperative $\mathrm{HB}$, the risk factor for transfusion and PMSA in primary TKA could be concordant. Our study confirmed that risk factors for PMSA in primary TKA were lower preoperative $\mathrm{HB}$ and more intraoperative blood loss.

Previous studies have used other methods in evaluating the cutoffs of preoperative $\mathrm{HB}$ to predict transfusion in TKA, but the cutoffs to optimal predict PMSA remained poorly understood. Maempel et al. identified threshold levels of $13.75 \mathrm{~g} / \mathrm{dL}$ for males and $12.75 \mathrm{~g} / \mathrm{dl}$ for females. The transfusion and complication rate increased significantly when preoperative was less than the threshold [9]. Yeh et al. found that preoperative HB cutoff values of $12.4 \mathrm{~g} / \mathrm{dL}$ for age above 70 and $12.1 \mathrm{~g} / \mathrm{dL}$ for age below 70 could be used to predict postoperative transfusion risk in TKA [10]. In another study, the authors showed that a preoperative $\mathrm{HB}$ value of $12.5 \mathrm{~g} / \mathrm{dL}$ was identified as the optimal cutoff for predicting postoperative transfusion across all patients with the administration of TXA [31]. Because the majority PMSA was moderate anemia in our study and preoperative HB threshold for moderate anemia $(80-109 \mathrm{~g} / \mathrm{L})$ was higher than that for transfusion $(<70$ or $70-100 \mathrm{~g} / \mathrm{L})[11,15]$, the reasonable hypothesis was that preoperative HB cutoff value to predict PMSA should be higher. Our study demonstrated the relationship between preoperative HB and PMSA firstly, finding that $131.5 \mathrm{~g} / \mathrm{L}$ for males and $138.5 \mathrm{~g} / \mathrm{L}$ for females were essential cutoff value in primary TKA in Chinese population. We provided an early warning value of preoperative HB for surgeons and patients.

In this study, we found that PMSA could lead to prolonged LOS and more expenses, which was consistent with previous studies. Abdullah et al. indicated that anemia was associated with prolonged LOS and increased perioperative transfusion [21]. Another study conducted by Kunz and his colleagues came to a similar conclusion [7]. In addition, substantial researches demonstrated that perioperative anemia could contribute to higher incidence of complication [5-7]. But the incidence of complications between PMSA and nonPMSA patients was comparable in our study. Probable seasons were as follow: firstly, the follow-up period was only 30 days, which was too short to monitor complete complications; secondly, the application of enhanced recovery protocols reduced the complication risk, decreasing the significance between the two groups [12]. Moreover, no patients needed blood transfusion in our study, indicating enhanced recovery protocols, particularly blood management strategies, played an important and beneficial role [3].

There are some limitations in this study. The amount of male cases is too small, only 84 patients (68 in nonPMSA and 26 in PMSA group), reducing the accuracy to identify the risk factors and predict preoperative HB cutoff value for PMSA. In addition, because most patients are discharged from hospital on POD3 and the blood samples are tested on POD1 and 3, we select the $\mathrm{HB}$ on POD1 and 3 as the diagnostic node for PMSA. It was reported that postoperative $\mathrm{HB}$ may continue to decline [32]. Thus, the incidence of PMSA is not accurate and we fail to monitor the comprehensive prevalence of anemia after primary TKA. Moreover, the follow-up time is only 30 days, leading to inability to evaluate the complications entirely, especially longterm complications, such as deep infection and impaired wound healing. At last, the limitation lies in its retrospective nature and the level of evidence is low. Further studies are requisite. We will conduct a randomized controlled study with longer follow-up time to investigate the relationship of preoperative $\mathrm{HB}$ and PMSA following joint arthroplasty in the near future.

\section{Conclusion}

In summary, lower preoperative $\mathrm{HB}$ and more intraoperative blood loss are independent risk factors for PMSA. Preoperative HB is a referable tool to predict PMSA. We provide an early warning value of preoperative $\mathrm{HB}$ for surgeons and patients undergoing primary TKA.

\footnotetext{
Abbreviations

TKA: Total knee arthroplasty; PMSA: Postoperative moderate and severe anemia; HB: Hemoglobin; AUC: Area under the receiver-operator curve; OR: Odds ratio; 95\% Cl: Confidence interval; TXA: Tranexamic acid; LOS: Longer length of stay; DVT: Deep venous thrombosis; PE: Pulmonary embolism; POD1: Postoperative day 1; POD3: Postoperative day 3; BMl: Body mass index.
} 


\section{Authors' contributions}

FXP designed the study. CGR and XLY were involved with data collection and manuscript preparation. $\mathrm{HX}, \mathrm{CY}, \mathrm{SYZ}$ and $\mathrm{ZYH}$ were involved with manuscript preparation. STQ, JNY and MLY assisted with data collection and data analysis. All authors read and approved the final manuscript.

\section{Funding}

Funded by the China Health Ministry Program (201302007) who had no involvement in study design, data collection, analysis or interpretation, drafting the manuscript or decision to submit it for publication.

\section{Declarations}

\section{Ethics approval and consent to participate}

This retrospective study was registered and approved by the Institutiona Review Board of our institution (2012-268). Written informed consent and research authorizations were obtained prior to surgery from all participants.

\section{Competing interests}

The authors declare that they have no competing interests.

\section{Author details}

'Department of Knee Injury (2), Luoyang Orthopedic Hospital of Henan Province, Orthopedic Hospital of Henan Province, Luoyang 471000, Henan Province, People's Republic of China. ${ }^{2}$ Department of Orthopaedic Surgery, West China Hospital, Sichuan University, No. 87 Guoxue Road, Chengdu 610041 Sichuan Province, People's Republic of China. ${ }^{3}$ Department of Orthopedics, The Third Hospital of Mianyang, Sichuan Mental Health Center, Mianyang 621000, Sichuan Province, People's Republic of China.

Received: 1 August 2021 Accepted: 13 September 2021

Published online: 26 September 2021

\section{References}

1. Kurtz $\mathrm{S}$, Ong K, Lau E, et al. Projections of primary and revision hip and knee arthroplasty in the United States from 2005 to 2030. J Bone Joint Surg Am. 2007;89(4):780-5.

2. Varghese VD, Liu D, Ngo D, et al. Efficacy and cost-effectiveness of universal pre-operative iron studies in total hip and knee arthroplasty. J Orthop Surg Res. 2021;16(1):536.

3. Wang D, Wang HY, Luo ZY, et al. Blood-conserving efficacy of multiple doses of oral tranexamic acid associated with an enhanced-recovery programme in primary total knee arthroplasty: a randomized controlled trial. Bone Joint J. 2018:100-B:1025-32.

4. Bailey A, Eisen I, Palmer A, et al. Preoperative anemia in primary arthroplasty patients-prevalence, influence on outcome, and the effect of treatment. J Arthroplast. 2021;36(7):2281-9.

5. Enokiya T, Hasegawa M, Morikawa Y, et al. Postoperative anaemia is a risk factor for bleeding-related event in thromboprophylaxis using fondaparinux sodium injection after total knee or hip arthroplasty. Biol Pharm Bull. 2020:43(2):266-71

6. Koch CG, Li L, Sun Z, et al. Magnitude of anemia at discharge increases 30-day hospital readmissions. J Patient Saf. 2017;13(4):202-6.

7. Kunz JV, Spies CD, Bichmann A, et al. Postoperative anaemia might be a risk factor for postoperative delirium and prolonged hospital stay: A secondary analysis of a prospective cohort study. PLOS ONE. 2020;15:e0229325.

8. Maradit Kremers H, Larson DR, Crowson CS, et al. Prevalence of total hip and knee replacement in the United States. J Bone Joint Surg Am. 2015;97(17):1386-97.

9. Maempel JF, Wickramasinghe NR, Clement ND, et al. The pre-operative levels of haemoglobin in the blood can be used to predict the risk of allogenic blood transfusion after total knee arthroplasty. Bone Joint J. 2016;98-B(4):490-7.

10. Yeh JZ, Chen JY, Bin Abd-Razak HR, et al. Preoperative haemoglobin cut-off values for the prediction of post-operative transfusion in total knee arthroplasty. Knee Surg Sport Traumatol Arthrosc. 2016;24(10):3293-8.

11. [Anonymous]. Nutritional anaemias. Report of a WHO scientific group. World Health Organization technical report series 1968; 405:5-37.
12. Ripolles-Melchor J, Abad-Motos A, Diez-Remesal Y, et al. JAMA Surg 2020;155:e196024

13. Cao G, Huang Q, Xu B, et al. Multimodal nutritional management in primary total knee arthroplasty: a randomized controlled trial. J Arthroplast. 2017:32(11):3390-5.

14. Zhang S, Huang Q, Xu B, et al. Effectiveness and safety of an optimized blood management program in total hip and knee arthroplasty: a large, single-center, retrospective study. Medicine. 2018;97(1):e9429.

15. Xie J, Ma J, Yao H, et al. Multiple boluses of intravenous tranexamic acid to reduce hidden blood loss after primary total knee arthroplasty without tourniquet: a randomized clinical trial. J Arthroplast. 2016;31(11):2458-64.

16. Dennis DA. Wound complications in TKA. Orthopedics. 2002;25(9):973-4.

17. Lasocki S, Krauspe R, von Heymann C, et al. PREPARE: the prevalence of perioperative anaemia and need for patient blood management in elective orthopaedic surgery: a multicentre, observational study. Eur J Anaesth. 2015;32(3):160-7.

18. Lee QJ, MakWP, Yeung ST, et al. Blood management protocol for total knee arthroplasty to reduce blood wastage and unnecessary transfusion. J Orthop Surg. 2015;23(1):66-70

19. Gu A, Malahias MA, Selemon NA, et al. Increased severity of anaemia is associated with 30-day complications following total joint replacement. Bone Joint J. 2020;102-B(4):485-94.

20. Abdullah HR, Ranjakunalan N, Yeo W, et al. Association between preoperative anaemia and blood transfusion with long-term functional and quality of life outcomes amongst patients undergoing primary total knee arthroplasty in Singapore: a single-centre retrospective study. Qual Life Res. 2019:28(1):85-98.

21. Abdullah HR, Sim YE, Hao Y, et al. Association between preoperative anaemia with length of hospital stay among patients undergoing primary tota knee arthroplasty in Singapore: a single-centre retrospective study. BMJ Open. 2017:7:e016403.

22. Biboulet $P$, Motais $C$, Pencole $M$, et al. Preoperative erythropoietin within a patient blood management program decreases both blood transfusion and postoperative anemia: a prospective observational study. Transfusion. 2020;60(8):1732-40.

23. Pennestrì F, Maffulli N, Sirtori P, et al. Blood management in fast-track orthopedic surgery: an evidence-based narrative review. J Orthop Surg Res. 2019;14(1):263.

24. Sinclair RC, Duffield KE, de Pennington JH. Improving preoperative haemoglobin using a quality improvement approach to treat iron deficiency anaemia. BMJ Open Qual. 2020;9(1):e000776.

25. Migliorini F, Maffulli N, Aretini P, et al. Impact of tourniquet during knee arthroplasty: a bayesian network meta-analysis of peri-operative outcomes. Arch Orthop Trauma Surg. 2021;141(6):1007-23.

26. Migliorini F, Maffulli N, Betsch M, et al. Closed suction drainages in Lower Limb Joint Arthroplasty: a level I evidence based meta-analysis. Surgeon. 2021;13:S1479-666X(21)00064-0.

27. Roberts M, Ahya R, Greaves M, et al. A one-centre prospective audit of periand postoperative blood loss and transfusion practice in patients undergoing hip or knee replacement surgery. Ann R Coll Surg Engl. 2000;82(1):44-8.

28. Erben HC, Hess F, Welter J, et al. Perioperative blood transfusions in hip and knee arthroplasty: a retrospective assessment of combined risk factors. Arch Orthop Trauma Surg. 2021. https://doi.org/10.1007/s00402-021-03763-w.

29. Huang Z, Huang C, Xie J, et al. Analysis of a large data set to identify predictors of blood transfusion in primary total hip and knee arthroplasty. Transfusion. 2018;58(8):1855-62.

30. Song K, Pan P, Yao Y, et al. The incidence and risk factors for allogenic blood transfusion in total knee and hip arthroplasty. J Orthop Surg Res. 2019;14(1):273.

31. Ryan SP, Klement MR, Green $\mathrm{CL}$, et al. Preoperative hemoglobin predicts postoperative transfusion despite antifibrinolytics during total knee arthroplasty. Orthopedics. 2019:42(2):103-9.

32. Gomez-Barrena E, Ortega-Andreu M, Padilla-Eguiluz NG, et al. Topical intraarticular compared with intravenous tranexamic acid to reduce blood loss in primary total knee replacement: a double-blind, randomized, controlled, noninferiority clinical trial. J Bone Joint Surg. 2014:96(23):1937-44.

\section{Publisher's Note}

Springer Nature remains neutral with regard to jurisdictional claims in published maps and institutional affiliations. 